\title{
Biomass Higher Heating Value Prediction Analysis by ANFIS, PSO- ANFIS and GA-ANFIS
}

\author{
Ceylan Z. ${ }^{1,4,}{ }^{*}$, Pekel E. ${ }^{2}$, Ceylan S. ${ }^{3}$ and Bulkan S. ${ }^{4}$ \\ ${ }^{1}$ Ondokuz Mayıs University, Faculty of Engineering, Industrial Engineering Department, 55139, Kurupelit Campus, Samsun/Turkey \\ ${ }^{2}$ Ondokuz Mayıs University, Faculty of Engineering, Computer Engineering Department, 55139, Kurupelit Campus, Samsun/Turkey \\ ${ }^{3}$ Ondokuz Mayıs University, Faculty of Engineering, Chemical Engineering Department, 55139, Kurupelit Campus, Samsun/Turkey \\ ${ }^{4}$ Marmara University, Faculty of Engineering, Industrial Engineering Department, 34722, Goztepe Campus, Istanbul/Turkey \\ Received: 20/05/2018, Accepted: 26/09/2018, Available online: 04/10/2018 \\ *to whom all correspondence should be addressed: e-mail: zeynep.dokumaci@omu.edu.tr \\ https://doi.org/10.30955/gnj.002772
}

\section{Abstract}

In this study, a new model for biomass higher heating value (HHV) prediction based on the Adaptive Neuro-Fuzzy Inference System (ANFIS) approach was proposed. Proximate analysis (volatile matter, fixed carbon and ash content) data for a wide range of various biomass types from the literature were used as input in model studies. Optimization of ANFIS parameters and formation of the model structure were performed by genetic algorithm (GA) and particle swarm optimization (PSO) algorithm in order to achieve optimum prediction capability. The best-fitting model was selected using statistical analysis tools. According to the analysis, PSO-ANFIS model showed a superior prediction capability over ANFIS and GA optimized ANFIS model. The Root Mean Square Error (RMSE), Mean Absolute Error (MAE), Mean Bias Error (MBE) and coefficient of determination $\left(R^{2}\right)$ for PSO-ANFIS were determined as $0.3138,0.2545,-0.00129$ and 0.9791 in the training phase and $0.3287,0.2748,0.00120$ and 0.9759 in the testing phase, respectively. As a result, it can be concluded that the proposed PSO-ANFIS model is an efficient technique and has potential to calculate biomass HHV prediction with high accuracy.

Keywords: Biomass, Higher Heating Value, Prediction, ANFIS, Genetic Algorithm, Particle Swarm Optimization

\section{Introduction}

Today, one of the most important global issues is enormous consumption of fossil fuels which are rapidly depleting and also causing environmental pollutions. To address this problem, many countries have begun to take advantage of alternative fuels in heat and electricity generation. Biomass is one of the most widely used alternative energy sources, and it is renewable, abundant, clean, and inexpensive. Utilizing biomass in a power plant or energy producing system requires knowledge of biomass characteristics, such as higher heating value (HHV).

HHV is the enthalpy of the combustion reaction of a fuel where carbon and hydrogen content are oxygenated to form to $\mathrm{CO}_{2}$ and $\mathrm{H}_{2} \mathrm{O}$. $\mathrm{HHV}$ is given at standard conditions $\left(101.3 \mathrm{kPa}, 25^{\circ} \mathrm{C}\right)$ and also contains the enthalpy value of water condensation. The $\mathrm{HHV}$ of a fuel component is a critical consideration for design, installment, and operation of power systems. The higher heating value can be determined by oxygen bomb calorimeter and is generally expressed in units of evolved energy per mass, such as $\mathrm{MJ} / \mathrm{kg}$, $\mathrm{kcal} / \mathrm{kg}$ or BTU/lbm. Oxygen bomb calorimeter is a consistent and simple way to measure HHV; however, it is not widely accessible in research laboratories because of its cost. Thus, in previous studies, various empirical equations have been suggested for higher heating value calculations based on ultimate composition or proximate analysis of results of fuel. However, these models have been proposed mainly for coal, and only a limited number are specifically for biomass fuel. Additionally, many of the previous models attempted to correlate the HHV with data from proximate and ultimate analyses. However, expensive elemental analysis equipment is needed for ultimate analysis to determine the final composition. Therefore, models that use proximate analysis results would be reasonable as it is convenient to use data obtained from a fast, easy and cheap way for a model to be formalized. Also, the heating value of fuels such as coal or biomass cannot be calculated simply from the formation enthalpies of $\mathrm{CO}_{2}$, $\mathrm{H}_{2} \mathrm{O}$ and other products because fuels are complex mixtures of compounds, and the associated bond energies cannot be obtained correctly. For instance, lignocellulosic biomass consists of different ratios of cellulose, hemicellulose, and lignin components. Since combustion is a complex process where numerous parallel reactions occur simultaneously, many unknown factors relevant to $\mathrm{HHV}$ are still unknown. Thus, modeling HHV is a challenging problem (Friedl et al., 2005; Akkaya, 2016; Suleymani and Bemani, 2018).

Most of the modeling studies on estimation of HHV have been based on calculations using linear regression method. However, the relationship between the higher heating value of biomass and the components of proximate analysis cannot be explained linearly. For this reason, 
estimation capability of linear regression models may be insufficient, especially when applied to a variety of samples (Akkaya, 2016). Therefore, in recent years, usage of artificial intelligence tools, which are widely applied to analyze, predict and describe a wide variety of nonlinear and complex systems, for the estimation of HHV has increased. Huang et al., (2008) employed Artificial Neural Network (ANN) to form models that enable estimation of HHV for straw biomass (Huang et al., 2008). They reported that the ANN model showed a good prediction capability. Uzun et al., (2017) applied different ANN models to predict HHV based on proximate analysis for a number of various biomass types and obtained an ANN model with high accuracy (Uzun et al., 2017). Ghugare et al., (2014) proposed a hybrid model of genetic programming and artificial intelligence in order to construct the biomass HHV prediction model (Ghugare et al., 2014). They reported that the results were better than the existing linear and/ or nonlinear equivalent values of the HHV prediction models. Ozveren (2017) studied HHV prediction using ANN models and reported that ANN is an efficient method for biomass HHV prediction (Ozveren, 2017). Estiati et al., (2016) studied on HHV estimation for various biomass types and revealed that ANN model gave better statistical results than linear and non-linear models (Estiati et al., 2016).

Nowadays, new models based on adaptive neuro-fuzzy inference system (ANFIS) have been used widely as an effective predictive tool for high order nonlinear functions due to its learning abilities which provide rapid adaptation to changes in systems. Fuzzy inference systems and artificial neural networks combined in ANFIS method are advantageous for estimation of parameters or describing system behavior with high accuracy (Austin and Amanollahi, 2016a; Barak and Sadegh, 2016; Dahmardeh Behrooz et al., 2017; Prasad et al., 2016; Rezakazemi et al., 2017). Akkaya, (2017) proposed ANFIS modeling for prediction of biomass HHV using proximate analysis and reported that ANFIS model was superior to existent models. As mentioned above, recently, different artificial intelligence tools have been employed to predict HHV. The proximate analysis data have been used as input parameters to enable a rapid, easy and low-cost estimation of the HHV where sophisticated and expensive equipment for experimental HHV measurement or ultimate analysis are not always available. The determination of the characteristics of the biomass is very important in terms of determining its fate.

HHV is one of the most important features of biomass, and its determination with high accuracy and low cost is important for potential applications of biomass. This study intends to obtain an improved correlation model for predicting the HHV of solid biomass by using their proximate analyses to reduce the need for time-consuming and high-cost ultimate analyses or oxygen bomb calorimeters. ANFIS method was applied to a biomass proximate analysis data set different containing many various species with different characteristics and a model for HHV estimation was established. The established model was improved by GA and PSO algorithms and models with high accuracy prediction capability were obtained. The performances of the models were evaluated using statistical tools and results are compared with previously reported models.

\section{Material and Methods}

\subsection{Dataset}

The aim of this study is to predict biomass HHV based on the proximate analysis results which can be obtained easily, rapidly and without needing expensive and sophisticated equipment. Thus, components of proximate analysis, namely volatile matter, fixed carbon and ash percentages are used as input parameters to obtain HHV as an output. To ensure that the proposed model has a wide range of validity, a data set containing biomass with different characteristics was created by reviewing the literature (Akkaya, 2016; Uzun et al., 2017; Zhang et al., 2017; Ghugare et al., 2014). Repeated samples were extracted from this data set and a simplified data set containing 353 samples was obtained. The volatile matter content of samples was ranged from approximately $13 \%$ to $91 \%$ by weight. Similarly, the fixed carbon range was found between $9 \%$ to $47 \%$. Ash content was between $0.2 \%$ and $40 \%$ (by weight). The output HHV of the biomass samples was 10 and $17 \mathrm{MJ} / \mathrm{kg}$.

\subsection{Adaptive Neuro-Fuzzy Inference System (ANFIS)}

Adaptive Neuro-Fuzzy Inference System (ANFIS) model combines both Fuzzy Inference System (FIS) and Artificial Neural Network (ANN) for solving complex and nonlinear problems. In this structure, the Takagi-Sugeno fuzzy system was employed as FIS. In the architecture used in this study, to simplify the model used, it is assumed that the framework of ANFIS includes two inputs $(x, y)$ and one output $(F)$. Thus, a fuzzy rule based on the Takagi-Sugeno type can be represented as below: (Rezakazemi et al., 2017; Ausati and Amanollahi, 2016b)

Rule 1:

If $\mathrm{x}$ is $A_{1}$ and $\mathrm{y}$ is $B_{1}$ then $\mathrm{F}_{1}=\mathrm{a}_{1} \mathrm{x}+\mathrm{b}_{1} \mathrm{y}+\mathrm{r}_{1}$

Rule 2:

If $\mathrm{x}$ is $A_{2}$ and $\mathrm{y}$ is $B_{2}$ then $\mathrm{F}_{2}=\mathrm{a}_{2} \mathrm{x}+\mathrm{b}_{2} \mathrm{y}+\mathrm{r}_{2}$

where, $A_{1}, A_{2}, B_{1}$ and $B_{2}$ are nonlinear parameters and membership functions for inputs ( $\mathrm{x}$ and $\mathrm{y}$ ) and $a_{1}, a_{2}, b_{1}, b_{2}$, $r_{1}$, and $r_{2}$ are linear, and output's (F) function parameters. The ANFIS architecture includes five layers with different functions. These layers can be named as a fuzzy layer, product layer, normalized layer, de-fuzzy layer and output layer, respectively. The function of each layer is depicted in Eqs. (3)-(8).

Each node ' $i$ ' in this layer produces a membership grade of a linguistic label. The membership relationship including the input and output functions of this layer can be written as: 
$F_{i}^{1}=\mu A_{i}(x) ; \quad i=1,2, \ldots$

$F_{i}^{1}=\mu B_{i}(y) ; \quad i=1,2, \ldots$

where $F_{i}^{1}$ and $F_{i}^{1}$ indicate the output functions and $\mu A_{i}(x)$ and $\mu B_{i}(y)$ shows membership functions.

Each node in this layer is a fixed node which calculates the 'firing strength' $\left(w_{i}\right)$ of each rule. In this second layer, output is the input signal's product that is referred to as:

$F_{i}^{2}=w_{i}=\mu A_{i}(x) \times \mu B_{i}(y), \quad i=1,2, \ldots$

where $F_{i}^{2}$ shows the output.

In layer 3, the weight function is under normalization as:

$F_{i}^{3}=w=\frac{w_{i}}{w_{1}+w_{2}}, \quad i=1,2, \ldots$

where $F_{i}^{3}$ was called normalized firing strength.

In layer 4, the output from the prior layer is multiplied with the Sugeno fuzzy rule's function:

$F_{i}^{4}=\bar{w}_{i} f_{i}=w_{i}\left(a_{i} x+b_{i} y+r_{i}\right), \quad i=1,2, \ldots$

where $F_{i}^{4}$ indicates the output of the layer 4 . In layer 5 , the sum of all outputs of every rule is computed and overall output can be calculated as follows:

$F_{i}^{5}=$ overall output $=\sum_{i} \bar{w}_{i} f_{i}=\frac{\sum_{i} w_{i} f_{i}}{\sum_{i} w_{i}}$

In the ANFIS structure, the first layer and the fourth layer include parameters that can be changed over time. The first layer contains the nonlinearities of the precursor parameters, while the fourth layer contains the linear result parameters. Both of these parameters can be modified and updated with a learning method that trains both of these parameters and also adapts to their conditions.

\subsection{Genetic Algorithm (GA)}

Genetic Algorithms ( $G A_{s}$ ) is an evolutionary heuristic search algorithm which depends on natural selection and genetic science. For this reason, it provides a random search that is used to solve optimization problems. Although random, GAs are by no means a coincidence. GAs use the available information to direct calls into the better performing region in the search area. The basic techniques of GA are designed to simulate the processes in natural systems, the evolution of which is necessary. In nature, competition between individuals for insufficient resources always leads to the emergence of the strongest individuals who dominate the weakest.

For a GA that represents a solution to a particular problem, there is a group of individuals within the search field. To continue the genetic analogy, these individuals resemble chromosomes and the variables represent genes. However, each solution is given an eligibility score that presents an individual's "competition" abilities (Rezakazemi et al., 2017; Anemangely et al., 2017).

\subsection{Particle Swarm Optimization (PSO) Algortihm}

Particle swarm optimization (PSO) is a population-based stochastic optimization technique. This algorithm was developed by inspiration from fish and insects moving in a swarm (Kennedy and Eberhart, 1995). The PSO optimization process begins with the selection of a population of random solutions or particles, and the generations are updated iteratively to seek optimization. Potential solutions, called particles in the PSO, pass through the problem area by following the best available solutions.

PSO has many similarities with evolutionary computing tools such as Genetic Algorithms (GA). However, unlike the GA, the PSO lacks evolutionist operators such as crossover and mutation. The advantage of the PSO is that it is easy to implement, and the number of parameters that need to be set is low. Optimization of different artificial intelligence tools with PSO has had successful applications in various areas.

\subsection{Evaluation of Model Performances}

The performances of models were evaluated according to the statistical tools such as root mean square error (RMSE), mean absolute error (MAE), mean bias error (MBE) and coefficient of determination $\left(R^{2}\right)$. The related equations for calculation of, RMSE, MAE and MBE and $R^{2}$, are given in Eqs. (9)-(12) as follows:

$$
\begin{aligned}
& \mathrm{RMSE}=\frac{1}{\mathrm{n}} \sum_{\mathrm{i}=1}^{\mathrm{n}} \sqrt{\left(\mathrm{HHV}_{\mathrm{i}}^{\text {actual }}-H H V_{i}^{\text {predicted }}\right)^{2}} \\
& M A E=\frac{1}{n} \sum_{i=1}^{n}\left|H H V_{i}^{\text {actual }}-H H V_{i}^{\text {predicted }}\right| \\
& M B E=\frac{1}{n} \sum_{i=1}^{n}\left(H H V_{i}^{\text {actual }}-H H V_{i}^{\text {predicted }}\right) \\
& \mathrm{R}^{2}=1-\frac{\sum_{\mathrm{i}=1}^{\mathrm{n}}\left(\mathrm{HHV}_{\mathrm{i}}^{\text {actual }}-\mathrm{HHV}_{\mathrm{i}}^{\text {predicted }}\right)^{2}}{\sum_{\mathrm{i}=1}^{\mathrm{n}} \sqrt{\left(\mathrm{HVV}_{\mathrm{i}}^{\text {actual }}-\mathrm{HHV}_{\mathrm{i}}^{\text {predicted }}\right)^{2}}}
\end{aligned}
$$

In these equations $\mathrm{HHV}_{\mathrm{i}}^{\text {actual }}, \mathrm{HHV}_{\mathrm{i}}^{\text {predicted }}$, and $\overline{\mathrm{HHV}_{\mathrm{i}}^{\text {predicted }}}$ represent the HHV data from literature, HHV data forecasted and the the mean predicted value for $i_{\text {th }}$ data point, respectively. Also, $\mathrm{n}$ denotes the total number of data points. The success of the model in representing the data set can be determined by examining $R^{2}$ which takes values between 0 and 1 , where values near 1 means $a$ better fit.

RMSE is used to determine the difference between the data of the prediction model and the actual data. MBE shows the general systematic error. The MAE measures the average size of errors in a series of estimates and represents a linear score, which means that all individual differences are weighted equally in the mean.

\section{Results and Discussion}

\subsection{Development of Models}

In this study, using the programming language of MATLAB 2017b software, ANFIS and two hybrid models of ANFIS, 
namely, GA-ANFIS, and PSO-ANFIS were proposed for biomass HHV prediction. In the first step of modeling, proximate analysis parameters (volatile matter, fixed carbon and ash) were set as input factors, and HHV was set as output factor.

In order to obtain enough prediction capability using ANFIS must be provided with an adequate number of clusters for the model. The developed ANFIS model uses fuzzy c-means clustering to form a fuzzy inference system. This process depends on the number of data that are evaluated for training. The rule extraction method first uses the Fuzzy cmeans (FCM) clustering function, known as genfis3, to determine the number of rules and membership functions for the antecedents and consequents. The Fuzzy c-means (FCM) clustering techniques (genfis3) were also used to optimize the result by extracting a set of rules that models the data and generate an initial FIS for ANFIS training. In order to construct a fuzzy system using ANFIS, the genfis3 function in MATLAB is first run to create a Sugeno-type FIS structure using fuzzy c-means clustering to extract a set of rules and membership functions that model the training data. This function allows the specification of the number of clusters used to model the data.

In order to increase the prediction capability of the ANFIS model, hybrid models were formed by using GA and PSO algorithms to optimize the ANFIS parameters. The flow chart indicating the procedures applied in model establishment for both GA-ANFIS and PSO-ANFIS are given in Figure 1 and 2 respectively.

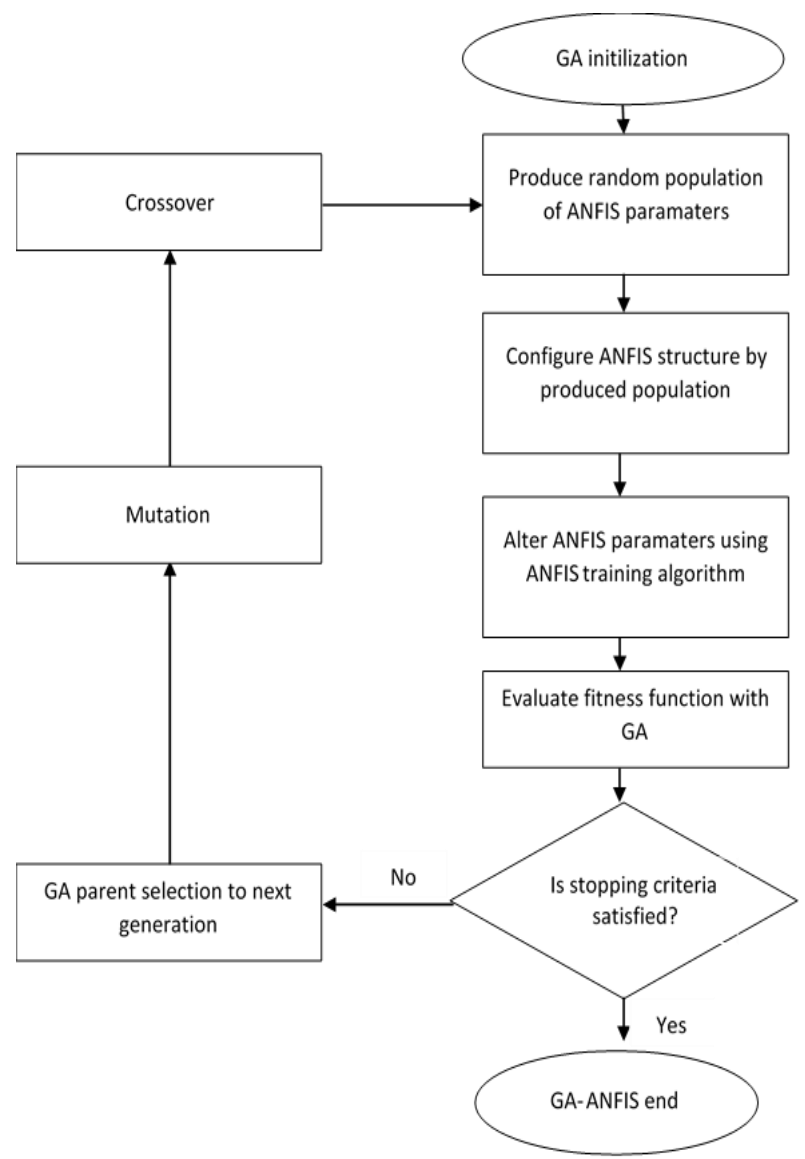

Figure 1. Scheme of the GA-ANFIS algorithm
In PSO-ANFIS, ANFIS provides the search space while PSO seeks the best solution by comparing objective function at each solution point. The difference between the actual data and the model output can be minimized by iterating the PSO algorithm (Sajjadi et al., 2017). The main benefit of the PSO are that this algorithm has a high degree of stability and does not depend on the derivative nature of objective function and can achieve to the optimal solution by tuning the membership functions.

In the current study, the Gaussian was applied as membership functions as recommended by many researchers. To develop the PSO-ANFIS, the optimum PSO parameters such as population size, maximum iterations number, initial inertia weight, inertia weight damping ratio and learning coefficients must be properly selected (Sajjadi et al., 2017). PSO-ANFIS model was applied by using a code was developed in MATLAB software. In GA-ANFIS, GA is combined with ANFIS to extend its prediction proficiency. GA-ANFIS method was also configured by coding in MATLAB to forecast biomass HHV. GA is implemented to improve ANFIS performance and minimize the error rates by tuning and optimizing the membership functions of a Sugeno type fuzzy inference system (Hossain et al., 2018). Gaussian shaped MFs were applied, which is recommended in previous studies. Table 1 presents both employed PSO and GA algorithm parameters in which stopping criteria only meet the number of iterations. These parameters are selected based on the authors' experiences within a trial-and-error process (Jiang et al., 2012; Rini et al., 2016; Chatterjee and Siarry, 2007).

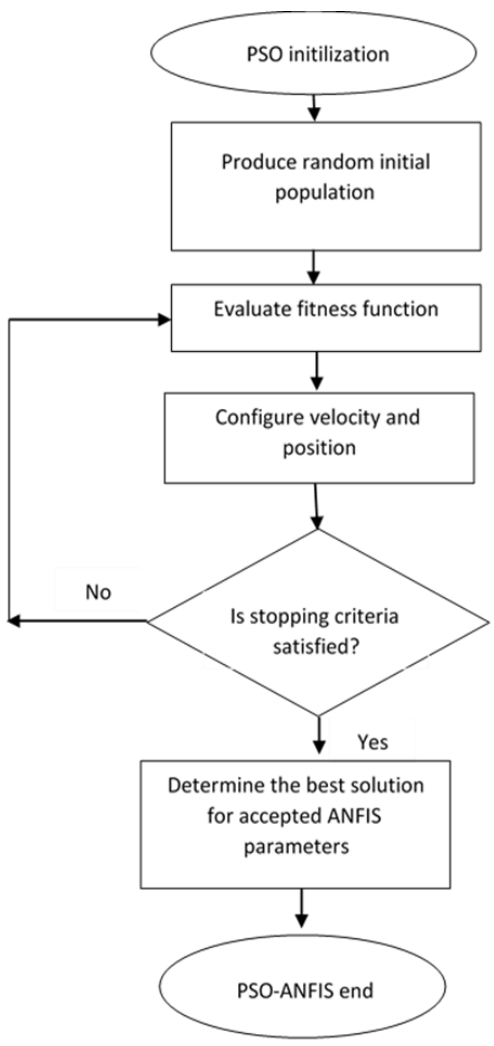

Figure 2. Scheme of the PSO-ANFIS algorithm 


\subsection{Evaluation of model performances}

All developed models utilize the training data for finding the relationships between the output HHV of biomass and the results of the proximate analysis. Afterward, they use the test data to check the accuracy of the model building. To fulfill this purpose, the available data on biomass proximate analysis is divided into two parts, with $70 \%$ (training with 247 data points) and 30\% (testing with 106 data points). Comparison of the models for RMSE, MAE, MBE and $R^{2}$ values can be observed clearly from Table 2 .
It can be noted that both GA and PSO algorithm can successfully improve the performance of the ANFIS model. The results of training and test analysis were given in Fig. 3 $a, b$ and $c$ for ANFIS, GA-ANFIS, and PSO-ANFIS respectively. To gain a better insight on prediction success of models, as depicted in Fig. 4, actual values were plotted against predicted ones and $\mathrm{R}^{2}$ was calculated (Suleymani and Bemani, 2018; Keybondorian et al., 2017; Akkaya, 2016).

Table 1. Details of the ANFIS, PSO ANFIS and GA-ANFIS for predicting HHV

\begin{tabular}{|c|c|}
\hline Parameter & Description/Value \\
\hline \multicolumn{2}{|l|}{ ANFIS model } \\
\hline Fuzzy structure & Sugeno-type \\
\hline Initial FIS for training & Genfis3 \\
\hline Maximumlterations Number & 1000 \\
\hline Number of fuzzy rules & 15 \\
\hline Input MF Type & Gaussian('gaussmf') \\
\hline Output MF Type & Linear \\
\hline Initial Step Size & 0.01 \\
\hline Step Size Decrease Rate & 0.9 \\
\hline Step Size Increase Rate & 1.1 \\
\hline \multicolumn{2}{|l|}{ PSO-ANFIS model } \\
\hline Population Size & 25 \\
\hline Maximum Iterations Number & 1000 \\
\hline Initial Inertia Weight ( $\left.W_{\min }\right)$ & 1 \\
\hline Inertia Weight Damping Ratio ( $\left.\mathrm{W}_{\text {damp }}\right)$ & 0.99 \\
\hline Personal Learning Coefficient 1 & 1 \\
\hline Global Learning Coefficient 2 & 2 \\
\hline \multicolumn{2}{|l|}{ GA-ANFIS model } \\
\hline Population Size & 25 \\
\hline Maximum Iterations Number & 1000 \\
\hline Crossover Percentage & 0.4 \\
\hline Mutation Percentage & 0.7 \\
\hline Mutation Rate & 0.15 \\
\hline Selection Pressure (beta) & 8 \\
\hline Gamma & 0.7 \\
\hline Selection Method & Roulette wheel \\
\hline
\end{tabular}

Table 2. Comparison between the performances of the developed models

\begin{tabular}{c|cccc|cccc}
\hline Model & \multicolumn{4}{|c|}{ Training data set } & \multicolumn{4}{c}{ Test data set } \\
\hline & ${ }^{a} \mathrm{RMSE}$ & ${ }^{\mathrm{b}} \mathrm{MAE}$ & ${ }^{\mathrm{c}} \mathrm{MBE}$ & ${ }^{\mathrm{d}} \mathrm{R}^{2}$ & ${ }^{\mathrm{a}} \mathrm{RMSE}$ & ${ }^{\mathrm{b}} \mathrm{MAE}$ & ${ }^{\mathrm{c}} \mathrm{MBE}$ & ${ }^{\mathrm{d}} \mathrm{R}^{2}$ \\
ANFIS & 0.5372 & 0.3774 & $3.4349 \mathrm{E}-07$ & 0.9339 & 0.6559 & 0.4465 & -0.01920 & 0.9228 \\
GA-ANFIS & 0.4347 & 0.3546 & -0.02950 & 0.9585 & 0.4504 & 0.3753 & -0.02950 & 0.9686 \\
PSO-ANFIS & 0.3138 & 0.2545 & -0.00129 & $\mathbf{0 . 9 7 9 1}$ & 0.3287 & 0.2748 & 0.00120 & $\mathbf{0 . 9 7 5 9}$ \\
\hline
\end{tabular}

${ }^{a}$ Root Mean Squared Error, ${ }^{b}$ Mean Absolute Error, ${ }^{c}$ Mean Bias Error, ${ }^{d}$ Correlation of Determination 

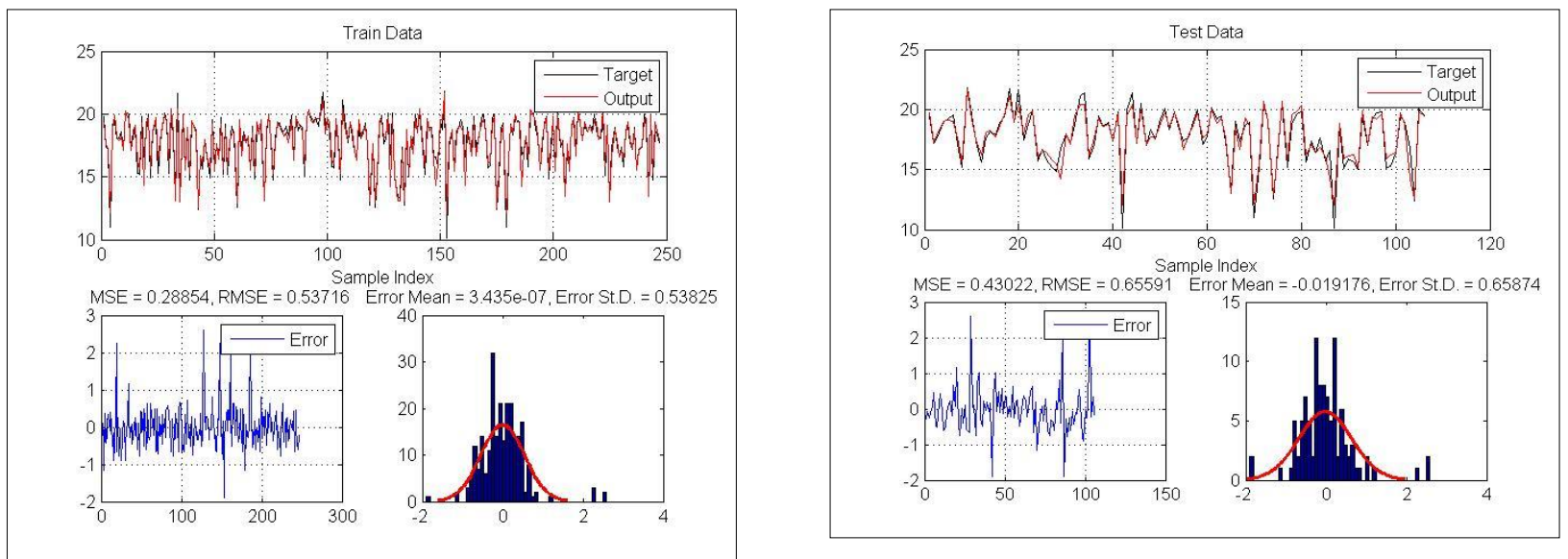

(a)
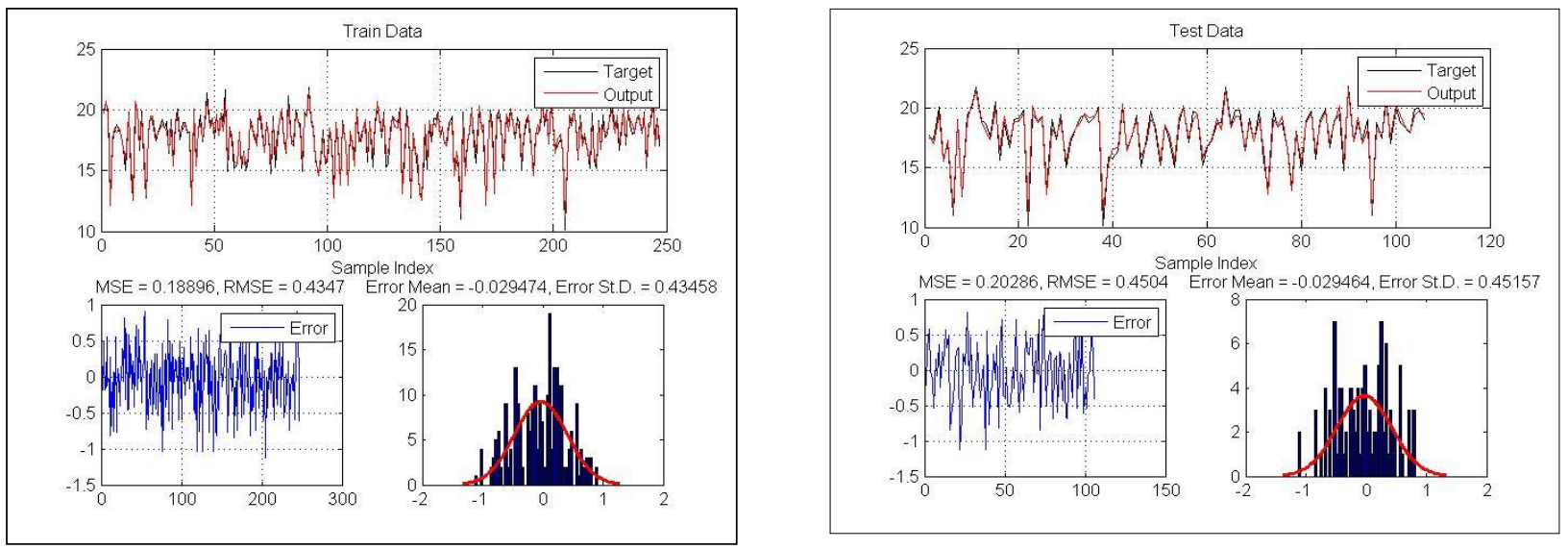

(b)
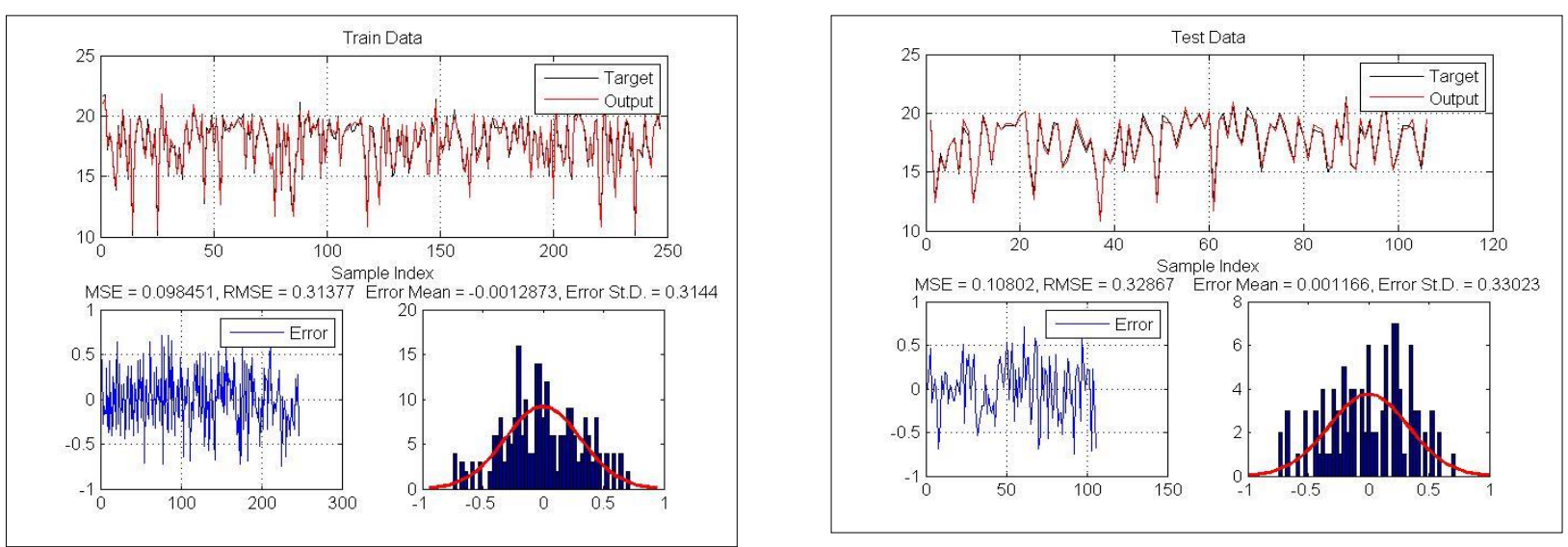

(c)

Figure 3. Training and testing results for a) ANFIS, b) GA-ANFIS, c) PSO-ANFIS 

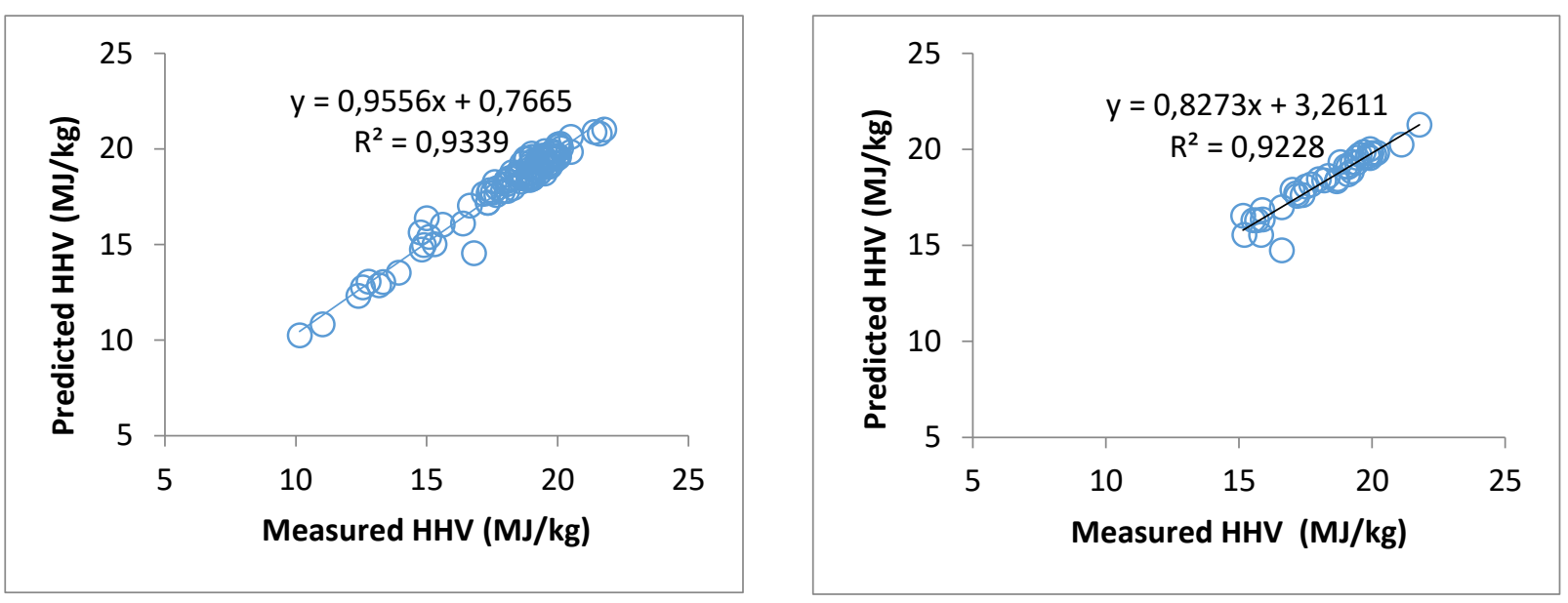

(a)
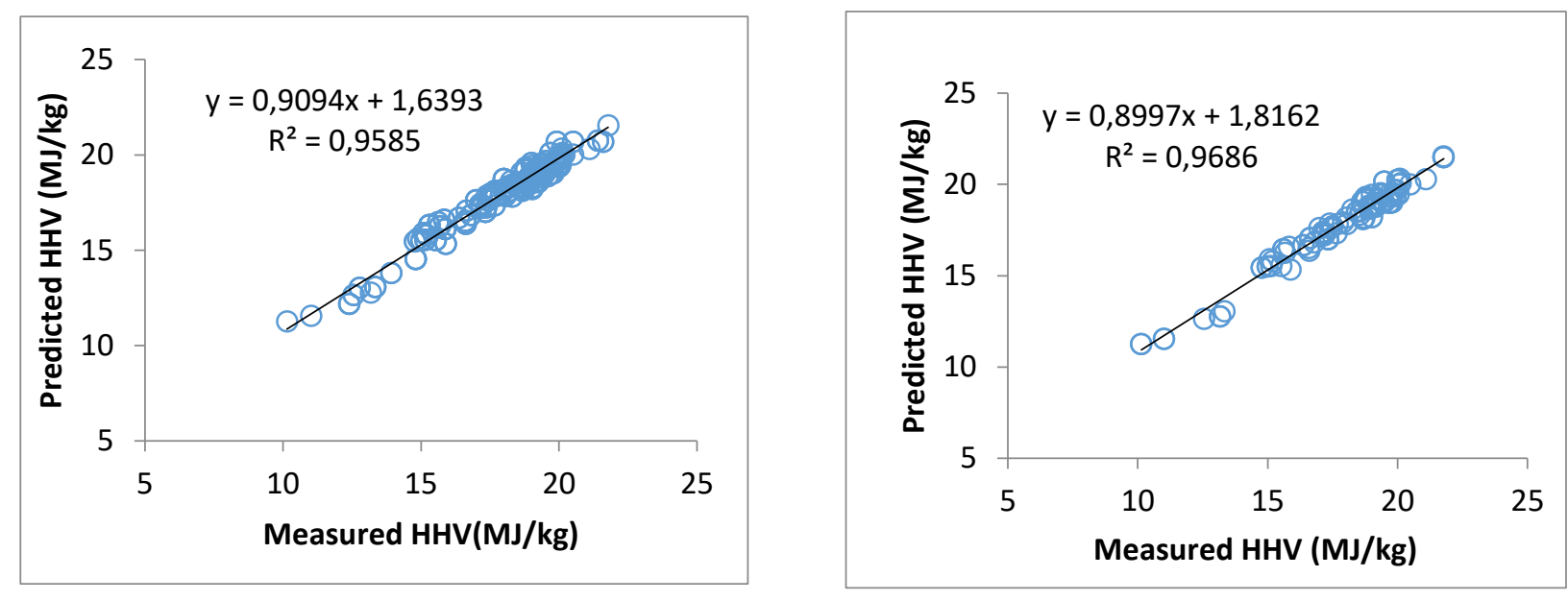

(b)
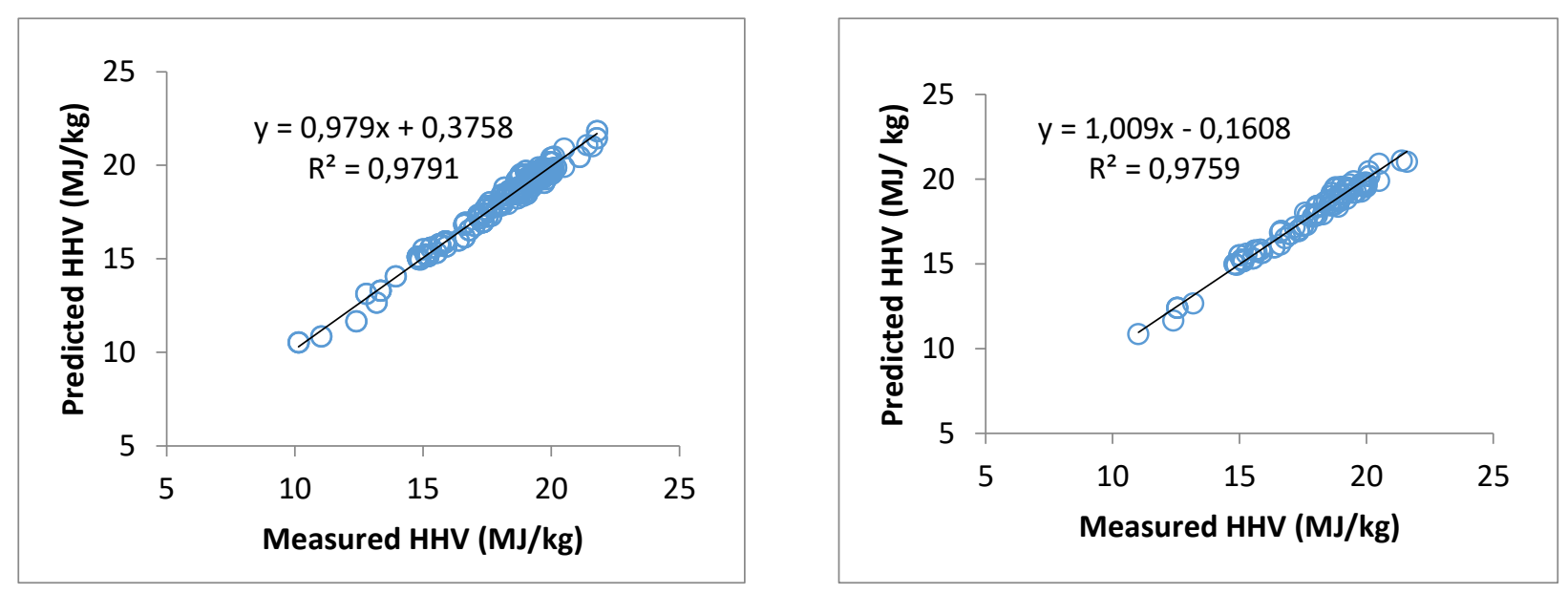

(c)

Figure 4. Predicted HHV data versus measured data for various models: (a) ANFIS, (b) GA-ANFIS, (c) PSO-ANFIS 
Table 3. Comparison of prediction capability of various models and developed PSO-ANFIS

\begin{tabular}{|c|c|c|c|c|}
\hline References & ${ }^{a} R M S E$ & ${ }^{b} M A E$ & ${ }^{c} M B E$ & ${ }^{d} R^{2}$ \\
\hline Yin et al., 2011 & 1.0970 & 0.8984 & -0.4332 & 0.8290 \\
\hline Nhuchhen and Salam, 2012 & 6.4262 & 2.94394 & -2.7172 & 0.2404 \\
\hline Callejon-Ferre et al., 2011 & 3.4707 & 2.2722 & 0.1707 & 0.3820 \\
\hline Ghugera et al., 2013 & 0.6123 & 0.4768 & -0.1408 & 0.9244 \\
\hline This study & 0.3287 & 0.2748 & 0.00120 & 0.9759 \\
\hline
\end{tabular}

${ }^{a}$ Root Mean Squared Error, ${ }^{b}$ Mean Absolute Error, ' Mean Bias Error, ${ }^{\mathrm{d} C o r r e l a t i o n ~ o f ~ D e t e r m i n a t i o n ~}$

The obtained correlation coefficient of the ANFIS, and GAANFIS and PSO-ANFIS models illustrate the predicted output HHV agrees well with the actual data. The correlation coefficient, which indicates the goodness of fit of the model, for the ANFIS, GA-ANFIS and PSO-ANFIS model are reported to be $0.9339,0.9585$ and 0.9791 for the training datasets, respectively. Similarly, PSO-ANFIS showed highest $R^{2}(0.9759)$ and lowest RMSE (0.3287), MAE (0.2748) and MBE (0.00120) values in testing dataset, too.

\section{Comparison with Literature}

As mentioned above the lowest statistical errors and the highest correlation coefficient were obtained with the PSOANFIS model. Therefore, this model was selected as the best model and compared with some commonly applied models given in literature. All models were applied to the current data set which was used in this study. The data set was kept constant and models were evaluated by using the same data set.

The results are shown in Table 3 and comparison depending on statistical analyzes was performed. Yin et al. (2011) investigated various linear equations to predict biomass HHV. Nhuchhem and Salam (2012) proposed a non-linear equation. Calejon-Ferre et al. (2011) proposed various linear and non-linear equations. Ghugare et al. (2014) used artificial intelligence tools and obtained best results with genetic programming optimized ANFIS model. It is obvious that the prediction capability of this model is higher than conventional linear or non-linear equation formalisms. As can be seen from the Table 3, PSO-ANFIS model showed higher $\mathrm{R}^{2}$ and lower RMSE, MAE and MBE values which indicate its potential as a prediction model for biomass HHV. This can be explained by the superiority of artificial intelligence tool in prediction over classical models.

\section{Conclusion}

The higher heating value (HHV) of biomass is of great importance in determining the potential of biomass and its possible application areas. In this study, the use of proximate analysis results which can be determined rapidly and cheaply, in the estimation of HHV of biomass has been investigated with artificial intelligence tools such as, ANFIS, GA-ANFIS, and PSO-ANFIS models. The main findings have been summarized as below:

- Extensive data consisting of 353 biomass from various geographies and of different characteristics were used.
- Biomass-specific ANFIS models were developed for predicting higher heating values.

- Hybrid GA-ANFIS, PSO-ANFIS models improved the prediction efficiency of the ANFIS model.

- The statistical parameters demonstrated that the PSO-ANFIS model has the best predicting performance.

- The literature comparison proved developed PSOANFIS model has a great potential.

- Developed models can help researchers in the design and operation of energy conversion systems.

\section{References}

Akkaya E. (2016), ANFIS based prediction model for biomass heating value using proximate analysis components, Fuel, 180, 687-693.

Anemangely M., Ramezanzadeh A., and Tokhmechi B. (2017), Shear wave travel time estimation from petrophysical logs using ANFIS-PSO algorithm: A Case Study from Ab-Teymour Oilfield, Journal of Natural Gas Science and Engineering, 38, 373-387.

Ausati S., and Amanollahi J. (2016), Assessing the accuracy of ANFIS, EEMD-GRNN, PCR, and MLR models in predicting PM2. 5, Atmospheric Environment, 142, 465-474.

Barak S. and Sadegh S.S. (2016), Forecasting energy consumption using ensemble ARIMA-ANFIS hybrid algorithm, International Journal of Electrical Power \& Energy Systems, 82, 92-104.

Chatterjee A. and Siarry P. (2007), A PSO-aided neuro-fuzzy classifier employing linguistic hedge concepts, Expert Systems with Applications, 33(4), 1097-1109.

Behrooz R.D., Esmaili-Sari A., Bahramifar N. and Kaskaoutis D.G. (2017), Analysis of the TSP, $\mathrm{PM}_{10}$ concentrations and watersoluble ionic species in airborne samples over Sistan, Iran during the summer dusty period, Atmospheric Pollution Research, 8(3), 403-417.

Estiati I., Freire F.B., Freire J.T., Aguado R. and Olazar M. (2016), Fitting performance of artificial neural networks and empirical correlations to estimate higher heating values of biomass, Fuel, 180, 377-383.

Friedl A., Padouvas E., Rotter H. and Varmuza K. (2005), Prediction of heating values of biomass fuel from elemental composition, Analytica Chimica Acta, 544(1-2), 191-198.

Ghugare S.B., Tiwary S., Elangovan V. and Tambe S.S. (2014), Prediction of higher heating value of solid biomass fuels using artificial intelligence formalisms, BioEnergy Research, 7(2), 681-692.

Hossain M., Mekhilef S., Afifi F., Halabi L.M., Olatomiwa L., Seyedmahmoudian M., Horan B. and Stojcevski A. (2018), Application of the hybrid ANFIS models for long term wind 
power density prediction with extrapolation capability, PloS one, 13(4), e0193772.

Huang C., Han L., Liu X. and Yang Z. (2008), Models predicting calorific value of straw from the ash content, International Journal of Green Energy, 5(6), 533-539.

Jiang H.M., Kwong C.K., Ip W.H. and Wong T.C. (2012), Modeling customer satisfaction for new product development using a PSO-based ANFIS approach, Applied Soft Computing, 12(2), 726-734.

Keybondorian E., Zanbouri H., Bemani A. and Hamule T. (2017), Application of MLP-ANN strategy to predict higher heating value of biomass in terms of proximate analysis, Energy Sources, Part A: Recovery, Utilization, and Environmental Effects, 39(22), 2105-2111.

Ozveren U. (2017), An artificial intelligence approach to predict gross heating value of lignocellulosic fuels, Journal of the Energy Institute, 90(3), 397-407.

Prasad K., Gorai A.K. and Goyal P. (2016), Development of ANFIS models for air quality forecasting and input optimization for reducing the computational cost and time, Atmospheric Environment, 128, 246-262.

Rezakazemi M., Dashti A., Asghari M. and Shirazian S. (2017), H2selective mixed matrix membranes modeling using ANFIS, PSO-ANFIS, GA-ANFIS, International Journal of Hydrogen Energy, 42(22), 15211-15225.

Rini D.P., Shamsuddin S.M. and Yuhaniz S.S. (2016), Particle swarm optimization for ANFIS interpretability and accuracy, Soft Computing, 20(1), 251-262.

Sajjadi B., Asaithambi P., Raman A.A.A. and Ibrahim S. (2017), Hybrid nero-fuzzy methods for estimation of ultrasound and mechanically stirring Influences on biodiesel synthesis through transesterification, Measurement, 103, 62-76.

Suleymani M. and Bemani A. (2018), Application of ANFIS-PSO algorithm as a novel method for estimation of higher heating value of biomass, Energy Sources, Part A: Recovery, Utilization, and Environmental Effects, 40(3), 288-293.

Uzun H., Yıldız Z., Goldfarb J.L. and Ceylan S. (2017), Improved prediction of higher heating value of biomass using an artificial neural network model based on proximate analysis, Bioresource technology, 234, 122-130.

Zhang Z.H., Hu M.G., Ren J., Zhang Z.Y., Christakos G. and Wang J.F. (2017), Probabilistic assessment of high concentrations of particulate matter (PM10) in Beijing, China, Atmospheric Pollution Research, 8(6), 1143-1150. 